\title{
Medios y prácticas periodísticas: Instagram como plataforma periodística. La experiencia de Agencia AUNO
}

\author{
Soledad Arréguez Manozzo \\ s.arreguez@gmail.com
}

Universidad Nacional de Lomas de Zamora.

María Emilia Merlo

memimerlo@gmail.com

Universidad Nacional de Lomas de Zamora.

DOI: https://doi.org/10.26422/aucom.2020.0901.arr

\begin{abstract}
Resumen
Las nuevas tecnologías de la información y la comunicación provocaron profundos cambios en el escenario mediático del siglo XXI. La expansión de Internet puso en crisis al esquema de broadcasting de los medios tradicionales. El modelo reticular de los 'nuevos medios' permitió la configuración de un nuevo entorno caracterizado por la multiplicidad de actores, medios y lenguajes, donde a su vez convergen numerosas voces, contenidos y prácticas.

En esta era digital, las redes sociales se convirtieron en uno de los principales medios por los que los ciudadanos acceden a los contenidos informativos: más de la mitad del planeta se encuentra en Internet y cada persona hace uso de medios digitales durante un promedio de 6 horas y 42 minutos al día, dándose la mitad de este tiempo de conexión a través de smartphones, según los datos recabados por Global Digital 2019 Report. De los tres mil cuatrocientos millones de usuarios de redes sociales en Internet, mil millones de ellos poseen cuentas activas en Instagram.

En este escenario las redacciones modificaron sus prácticas y rutinas periodísticas, con el fin de acercar los contenidos informativos a una mayor audiencia. Instagram, como otras herramientas del mundo móvil, se presenta como un espacio emergente de innovación periodística por su versatilidad, impacto visual y capacidad de transmitir información en directo. Particularmente el uso de stories de Instagram representa una gran oportunidad para alcanzar una audiencia joven, brindando información en tiempo real y contextualizada, y fomentando la participación. A lo largo de este trabajo expondremos la experiencia de Agencia AUNO en la aplicación de Instagram para el trabajo periodístico en un nuevo entorno mediático. En ese sentido implementamos las funcionalidades de Instagram para realizar coberturas periodísticas y generar nuevas formas de relacionarnos con nuestra comunidad. Si bien el uso de la plataforma es trans-
\end{abstract}


versal a todas las temáticas, su empleo resulta especialmente provechoso en coberturas de temas de sociedad y géneros donde los recursos de la propia herramienta nos permiten enriquecer el contenido y el storytelling de las coberturas.

Palabras clave: redes sociales, Instagram, audiencias, Agencia AUNO.

\section{Instagram as journalistic platform. The case of Agencia AUNO}

\section{Abstract}

The new information and communication technologies cause big changes in the media scene in the 21st century. The expansion of the Internet put the traditional media transmission scheme in crisis. The reticular model of the "new media" enabled the configuration of a new environment determined by the multiplicity of actors, media and languages, where sources, contents and narrativa practices converge.

In this digital age, social networks became one of the main means by which citizens access informational content: more than half of the planet is on the Internet and each person makes use of digital media for an average of 6 hours and 42 minutes a day, giving half of this connection time through smartphones, according to Global Digital 2019 Report. Of the three thousand four hundred million users of social networks on the Internet, millions of them have active accounts on Instagram.

In this scene, the newsrooms modified their journalistic practices and routines, in order to bring the informative contents closer to a larger audience. Instagram, like other apps in the mobile world, is presented as an emerging space for journalistic innovation due to its versatility, visual impact and ability to transmit information live. Particularly, the use of Instagram stories represents a great opportunity to reach a young audience, providing real-time and contextualized information, and encouraging participation.

Throughout this document we explain the experience of the AUNO Agency in the Instagram application for journalistic work in a new media environment. In that sense, we implement Instagram functionalities to do media coverage and generate new ways of relating to our community. Although the use of the platform is transversal to all the themes, its use is especially beneficial in media coverage of society and gender issues where the resources of the app itself allow us to enrich the content and the storytelling.

Keywords: Social Networks, Instagram, Audiences, Agencia AUNO.

\section{Instagram como plataforma jornalística. A experiência da Agência} AUNO

\section{Resumo}

As novas tecnologias da informação e comunicação provocaram profundas mudanças na esfera midiática do século XXI. A expansão de Internet levou a crise do esquema de broadcasting da mídia tradicional. O modelo reticular de "novas mídias" permitiu a configuração de um novo contexto caracterizado pelos múltiplos atores, mídias e linguagens, onde ao mesmo tempo convergem muitas vozes, conteúdos e práticas.

Nesta era digital, as redes sociais viraram um dos principais canais pelos quais os cidadãos acessam aos conteúdos informativos: mais da metade do planeta está na Internet, e cada pessoa faz uso de mídias digitais em uma média de 6 horas e 42 minutos ao dia, sendo a metade deste tempo conexões através de smartphones, segundo os dados de Global Digital 2019 Report. Dos três 


\begin{abstract}
mil e quatrocentos milhões de usuários de redes sociais na Internet, mil milhões deles possuem contas ativas no Instagram.

O cenário leva as redações mudarem suas práticas e rotinas jornalísticas, com a finalidade de apresentar os conteúdos informativos para uma audiência maior. Instagram, junto a outras ferramentas do mundo móvel, se estabelece como um espaço emergente de inovação jornalística pela sua versatilidade, impacto visual e capacidade de transmitir informação ao vivo. $\mathrm{O}$ uso da ferramenta de stories de Instagram é uma grande oportunidade para chegar á uma audiência jovem, brindando informação em tempo real e contextualizada, fomentando a participação.

O presente documento expõe a experiência da Agência AUNO na aplicação do Instagram no trabalho jornalístico em um novo entorno midiático. Neste sentido, colocamos em prática as funcionalidades do Instagram para desenvolver coberturas jornalísticas e novos jeitos de relacionamento com nossa comunidade. $\mathrm{O}$ uso da plataforma é transversal a todas as temáticas, porém seu uso resulta especialmente proveitoso em temáticas sociais e de gênero, onde os recursos da própria ferramenta permitem enriquecer o conteúdo e storytelling das coberturas.
\end{abstract}

Palavras chave: Redes Sociais, Instagram, Audiências, Agencia AUNO.

\title{
Introducción
}

La red de redes revolucionó gran parte de los paradigmas que explicaban la comunicación pública en los medios masivos. La llegada de Internet, y más tarde del ciberespacio, modificó el esquema mediático del siglo XX regido por el modelo de broadcasting. La prensa gráfica, las cadenas de televisión y las emisoras practicaban un modelo lineal y asimétrico, en el que, como únicos emisores, transmitían los mensajes a un conjunto de receptores, que se caracterizaban por ser pasivos, con poca posibilidad de interactuar con aquellos que construían los mensajes (Arréguez, Fernández, 2019).

Como señala Ramonet (2011), los medios tradicionales entraron en crisis a partir del "meteorito" Internet, que provocó "un cambio radical de todo el ecosistema mediático" por lo que la propia práctica periodística "debe ser reconstruida y reinventada" (Ramonet, 2011 p. 11). El periodismo atraviesa desde entonces una crisis de identidad al haber perdido el monopolio de la información en la sociedad, ya que el escenario se invierte: se pasa de medios de masas a masas de medios.

Con la red, los mass media dejaron de ser los únicos emisores, ya que comenzaron a aparecer en el terreno comunicacional nuevos sitios, bitácoras y publicaciones en línea. En la sociedad de masas existían unos pocos "medios sol" que, como centro del sistema, determinaban los flujos de información y de comunicación. Con la emergencia y expansión de los nuevos medios, se deja atrás el sistema medio-céntrico a uno yo-céntrico, en el que los usuarios tienen la posibilidad de publicar textos, subir fotos y videos, compartir contenidos.

Estas nuevas formas de comunicación de los nuevos medios se caracterizan por su transformación tecnológica (digitalización), una configuración muchos-a-muchos (reticularidad), estructuras textuales no secuenciales (hipertextualidad), la convergen- 
Soledad Arréguez Manozzo, María Emilia Merlo

Medios y prácticas periodísticas: Instagram como plataforma periodística.

La experiencia de Agencia AUNO

cia de medios y lenguajes (multimedialidad) y la participación activa de los usuarios (interactividad) (Scolari, 2008).

El desarrollo de la World Wide Web, y en particular, la web 2.0, permitió la creación y expansión de comunidades de usuarios y de servicios orientados a la creación, publicación y distribución de contenidos. En la sociedad de redes cada ciudadano es un "periodista en potencia", ya que, desde su computadora, tableta o smartphone, el internauta utiliza las herramientas 2.0 para producir y compartir contenido y así informar al mundo (Ramonet, 2011). En esta segunda generación de la web prima el intercambio y la colaboración entre los internautas.

En la era postbroadcasting, los usuarios adquieren una identidad digital: se comunican, compran, trabajan, se entretienen y se informan mediante las herramientas y aplicaciones de Internet. Los internautas se convirtieron en prosumidores -productores y consumidores de contenidos- que pasan cada vez más tiempo en la web. La participación de los nuevos usuarios en el esquema comunicacional significó la circulación de contenidos sin sumisión a estructuras jerárquicas ni dependencia a los canales tradicionales de comunicación. Tal como señala Denis de Moraes, sobre las mutaciones comunicacionales en el ciberespacio:

\begin{abstract}
La pragmática de Internet deshace la polaridad entre un centro emisor activo y receptores pasivos. Las interfases tecnológicas instituyen un espacio de transacción cuyo soporte técnico, en proceso constante, proporciona comunicaciones intermitentes, precisas y ultrarrápidas en una interacción entre todos y todos y no más entre uno y todos (De Moraes, 2004, párr. 2).
\end{abstract}

Estamos en un entorno en el que hay más medios sociales, más actores y más información. Sin duda, el modo de dar tratamiento a las noticias se ve influenciado por las mutaciones del esquema de medios, como así también por los nuevos hábitos de los usuarios. Inmersos en la cultura de la convergencia, los ciudadanos tienen acceso a la noticia y de alguna manera, son "socios de la autoría", ya que el contenido "gana espacio en las redes sociales a partir de estos actores sociales, a menudo acompañados de algún comentario sobre el tema que reproduce algo personal del prosumidor" (Campalans, Renó, Gosciola, 2014, p. 57).

\title{
Las redes sociales como canal y fuente
}

Como señala la holandesa José Van Dijck (2016), las tecnologías de comunicación evolucionaron como parte de las prácticas sociales cotidianas, y los nuevos medios interactivos han moldeado nuevas formas de sociabilidad. Los usuarios se volcaron a las plataformas sociales, como Facebook, que se convirtieron en "fuerzas fundamentales para la construcción de la socialidad" en la era 2.0. 
Si bien las redes sociales se presentan como plataformas para el intercambio entre usuarios, las empresas periodísticas vieron la oportunidad de sumarse a ese muro de amigos. Los medios de comunicación crearon perfiles sociales y buscaron utilizar estos espacios como canales de distribución de sus contenidos, entendiendo que cada vez la audiencia pasa tiempo allí. Además, son utilizados por los periodistas como fuente, dado que cada vez más entidades, colectivos y personas se integran a la red, y realizan allí sus reclamos, anuncios o pedidos.

Asimismo, los medios buscan que los usuarios formen parte de sus comunidades en línea y que interactúen con sus contenidos ya sea haciendo comentarios, compartiendo material y/o generando uno nuevo.

Desde la Agencia Universitaria de Noticias y Opinión (AUNO) de la Facultad de Ciencias Sociales de la Universidad Nacional de Lomas de Zamora (UNLZ), espacio de práctica profesional para las carreras de Periodismo y Comunicación Social, tomamos el desafío de tener presencia en estas plataformas. Esto derivó en un ciclo de capacitaciones para estudiantes, docentes y graduados sobre Periodismo Digital y Community Management y, sobre todo, en la conformación de un equipo de Social Media de la agencia, cuya tarea estuviera orientada a pensar nuevas narrativas periodísticas, acompañadas de nuevas rutinas periodísticas. Como señala Orihuela, el nuevo escenario de la comunicación pública es la ocasión "para redefinir el perfil y las exigencias profesionales de los comunicadores” (Orihuela, 2002).

\section{Instagram, el álbum compartido}

Instagram es una red social y aplicación para sistemas IOS y Android que fue creada en 2010 por Kevin Systrom y Mike Krieger. Pertenece al género de fotografía y video, permite publicar varios tipos de contenido, incluyendo fotos, videos breves y stories.

La aplicación posee un feed en el que aparecen todas las publicaciones (fotos y vídeos) de las cuentas que se siguen, en tanto que el feed de un perfil es la página en la que aparecen todas las publicaciones de un solo perfil. La aplicación tiene la opción de sumar más de una foto a la publicación, y también da la posibilidad de realizar collages de fotos para publicar en la red social. Una vez elegida la o las imágenes a publicar, Instagram da la opción de utilizar filtros. Cada filtro de Instagram tiene su propia personalidad (color, tono y luz) para cambiar nuestra foto, y permite destacar ciertos aspectos de esta. También existe la opción de edición en tanto a tono, luz, color y enfoque, que permite una customización aún mayor de la imagen.

La aparición de Instagram Stories en 2010 representó una disrupción total en el recorrido de la plataforma, que sufría una merma en la cantidad de nuevos usuarios frente a la red social Snapchat, aplicación de la cual recoge la idea de implementar ese 
Soledad Arréguez Manozzo, María Emilia Merlo

Medios y prácticas periodísticas: Instagram como plataforma periodística.

La experiencia de Agencia AUNO

"segundo timeline", con la efimeridad y brevedad como sus postulados de base. Lo que comenzó como una estrategia para no perder al público joven inauguró una nueva forma de contar en Instagram. Rápidamente las historias instituyeron modos de narrar propios que acabarían creando un estilo reconocible que los medios tradicionales y otras RRSS buscarían imitar.

Las stories son una funcionalidad de Instagram que permite a los usuarios compartir fotos y vídeos efímeros que desaparecen a las 24 horas. Estas historias permiten subir, en formato vertical, fotografías y vídeos de hasta 15 segundos de duración, y personalizarlos con filtros, stickers, GIF, etiquetas de ubicación y hashtags. Estas piezas están más vinculadas a la instantaneidad y la espontaneidad, ya que se utiliza para mostrar elementos sin que aparezcan en el feed del perfil. También presentan una clara vertiente interactiva, pues ofrecen como posibilidad la publicación de encuestas, trivias y valoraciones -predeterminadas o personalizables- que invitan a la audiencia a expresarse sobre alguna temática particular o bien retroalimentar la información brindada con sus experiencias y apreciaciones personales.

La plataforma también ofrece el canal IGTV que permite a los usuarios ver videos de formato largo y vertical. La diferencia entre Instagram Live e IGTV es que la primera está filmada y subida en vivo, mientras que la segunda es material ya grabado, por lo tanto, permite la edición.

\section{Quiénes están en Instagram}

En junio de 2018, Instagram anunció que había pasado la marca de los mil millones de 'cuentas activas', aunque aclaró que esta cifra no representaba a usuarios únicos. De acuerdo con el Digital Report 2019, las últimas cifras de audiencia publicitaria de la plataforma muestran un crecimiento que alcanza una audiencia publicitaria de 895 millones de usuarios activos en todo el mundo en el momento de la redacción.

Según Hootsuite, la audiencia gruesa de Instagram se enmarca en el rango etario de 18 a 34 años, los llamados millenials, que se identifican por pasar gran parte del día en línea y utilizar las redes sociales para informarse, sobre medios clásicos como diarios, radio y televisión. ¿Qué diferencial representan para este grupo las historias? Básicamente, su capacidad de síntesis, su renovación constante, a tono con las temáticas virales de la red y la interpelación directa de estas hacia su universo narrativo, así como el "miedo a quedarse afuera", más conocido como FOMO (DeHaan, Gladwell, Murayama, Przybylski, 2013). Este término describe la sensación de malestar que los usuarios pueden llegar a sentir al saber que otras personas están realizando actividades agradables y uno no forma parte de ello. Los afectados por el FOMO temen quedar al margen, sentirse excluidos por no haber estado pendientes de lo que sucede en redes. 
Frente a las últimas actualizaciones de la plataforma y los nuevos fenómenos relacionados a la celeridad de su joven audiencia, visualizamos la oportunidad de hacer uso de nuestra cuenta de Instagram como un espacio de comunicación responsable y alternativa, desde donde reconfigurar y reconvertir los tradicionales esquemas periodísticos de selección y elaboración de las noticias. Asimismo, planeamos la apertura a nuestros seguidores de forma tal que nuestro objetivo último fuera la construcción de comunidad desde el medio universitario.

\section{El potencial de Instagram para el periodismo}

Instagram es una plataforma que permite contar de forma visual, conversar con los usuarios, y explota la capacidad de expandir historias y crear significado a través del transmedia storytelling.

El término transmedia storytelling o narrativa transmediática fue acuñado en 2003 por Henry Jenkins en un artículo de Technological Review del MIT (Jenkins, 2003, párr.2). El concepto refiere a un "proceso en elementos integrantes de una ficción se dispersen de forma sistemática a través de múltiples canales de distribución con el fin de crear una experiencia de entretenimiento unificada y coordinada" (Jenkins, 2003).

En la narrativa transmedia, es posible aprovechar "las posibilidades comunicacionales presentes en la sociedad posmoderna, en la que la movilidad y la liquidez de estructuras, o sea, la interactividad, asumen papeles importantes en el campo de la comunicación, como la de involucrar y atraer al receptor para la interpretación participativa del mensaje" (Porto y Flores; 2012).

Entendemos que en la construcción de una narrativa periodística posible se deben tener en cuenta las posibilidades que brindan el feed y las stories. El feed es lo primero que los seguidores ven al entrar a un perfil, por lo que un feed ordenado y armonioso desde lo estético, pero también desde lo discursivo, genera buena impresión desde el primer momento y es fundamental para obtener más seguidores. En el feed se suelen encontrar las fotos más producidas, mientras que las stories de carácter efímero pueden ser un poco más "naturales" y "caseras". Estas particularidades técnicas nos interpelan a tomar decisiones editoriales sobre qué tipo de contenido se generará para cada uno de estos espacios.

\section{La implementación de Instagram en Agencia AUNO}

La Agencia Universitaria de Noticias y Opinión (AUNO) de la Facultad de Ciencias Sociales de la Universidad Nacional de Lomas de Zamora (UNLZ) incorporó su cuenta en Instagram en 2015. Este perfil se sumó a los que el medio ya tenía en las redes sociales Facebook y Twitter. 
Soledad Arréguez Manozzo, María Emilia Merlo

Medios y prácticas periodísticas: Instagram como plataforma periodística.

La experiencia de Agencia AUNO

En un principio, la plataforma se pensó como un espacio para construir comunidad en torno al espíritu "aunero", entendido como el sentimiento de pertenencia de los estudiantes de Periodismo y Comunicación Social que han pasado desde 1990 por el espacio de práctica profesional. En ese sentido, las primeras publicaciones apuntaban a resaltar la identificación con la universidad, la facultad y el espacio de la agencia de noticias, que actúa como dispositivo pedagógico y como medio de comunicación regional. La narrativa estaba acompañada por emojis de indicación, como manos y flechas y los hashtags de posicionamiento como \#AUNO \#AgenciaUniversitariaDeNoticiasYOpinión \#UNLZ \#SocialesUNLZ.

El primer protocolo de publicación de la red establecía publicaciones de texto con imágenes, una periodicidad de tres a cinco veces por semana, una banda horaria por la mañana, de 8 a 11, y otra por la noche, de 19 a 22.

En una segunda etapa, las publicaciones abordaban noticias del ámbito universitario y de la región. Los posteos se caracterizaron por ser noticias con impacto emocional que se pudieran contar en pocos caracteres y que generaran interés en el usuario para que accediera al sitio web. En ese sentido, abundaban las temáticas deportivas, dado que en la localidad hay varios clubes de fútbol de diferentes ligas. En esa etapa hubo una mayor relación con el sitio web, y la periodicidad de las publicaciones comenzó a variar según su actualización. En cuanto a la narrativa de la plataforma, se establecieron hashtags y emojis determinados por sección y localidad o zona geográfica de la noticia.

Desde 2018, iniciamos una tercera fase de producción de contenidos orientados a producir información sin seguir el ritmo de la redacción. Podemos decir que concebimos la cuenta de IG como un medio en sí mismo: con su propia estética, dinámica, agenda y rutina periodística. La cuenta de Instagram comenzó a producir contenido nativo, sin necesidad de seguir la agenda informativa del sitio web de la Agencia, lo que brindó libertad en tanto a la elección de temas y la forma de estructurarlos, pudiendo adoptar un lenguaje propio y diseño diferenciado.

Esta decisión nació como solución para la cobertura rápida de fenómenos virales y desde una perspectiva visual, que no dependiera de las rutinas de trabajo de la redacción. El equipo a cargo de Instagram decidió entonces realizar un periodo de monitoreo de la cuenta, a fines de obtener información para conocer a la audiencia de seguidores, sus intereses y especificidades de consumo. Con ese diagnóstico, se procedió a delimitar una paleta de colores, emojis, y etiquetas a emplear en los posteos feed, mientras que se eligió a una de las estudiantes/redactoras para encarnar la cara de las historias y de IGTV. Su labor consiste en presentar la información desde un lugar de interpelación, coloquial y cercano, invitando a opinar, a la retroalimentación conversacional típica de la herramienta. 
Austral Comunicación

Volumen 9, número 1 (Junio de 2020): 1-12. ISSN 2313-9137 E

Nuestro criterio a la hora de elegir los temas se guía por los trendings topics o novedades de la semana, aunque también poseemos una agenda propia de efemérides y conmemoraciones locales, porque sabemos que los temas zonales tratados desde una perspectiva cercana e íntima es un diferencial para nosotros frente a otros medios zonales. Mantenemos de forma constante un monitoreo del desempeño de las distintas temáticas tratadas y eso nos define a la hora de darle continuidad o actualización a algún fenómeno, aunque no nos movemos "por los likes", nos ayuda a entender por qué un contenido tuvo mejor desempeño que otro e identificar los aspectos que suman a la narrativa, para poder replicarlos y adaptarlos a otros contenidos.

Más allá de los números arrojados por las métricas de Instagram, otra de las tareas que el equipo de monitoreo y gestión realiza es el análisis de los comentarios y mensajes directos, ya sea en cuanto a valoraciones u opiniones de la audiencia acerca del material publicado, como también contemplando su interés en el seguimiento de algún acontecimiento particular, la ampliación de historias o la búsqueda de información extra sobre lo abordado.

\section{Experiencias exitosas}

En estos primeros pasos en la red hemos logrado cubrir hechos periodísticos de un modo diferente, con la mirada en la dinámica de la red y en nuestra audiencia. En este sentido podemos destacar la cobertura que se realizó en torno a los reclamos de los movimientos feministas y las temáticas de géneros y diversidades.

Detectamos en nuestra comunidad una preferencia por temáticas vinculadas con asuntos de sociedad y género que posean impronta local, por lo que apostamos a cubrir este tipo de eventos. Como resultado, podemos decir que nuestros seguidores valoraron la capacidad expresiva y espontánea que ofreció la plataforma a la hora de cubrir las jornadas de debate en el Congreso por el Aborto Legal en 2018, que fue uno de los acontecimientos culmines de interacción y engagement para la cuenta. Nuestro foco estuvo puesto en la crónica visual a través de las historias, apuntando a registrar tanto las exposiciones de los legisladores como las actividades de la "marea verde" en las calles.

Nuestra audiencia demostró predilección por temas de coyuntura social con enclaves locales, como lo fueron las protestas por los tarifazos durante 2018 y 2019 en Lomas de Zamora, o el reclamo del aborto legal, cobertura de la cual uno de sus puntos fuertes fue la vertiente local (entendida en el despliegue de actividades como "trenazos", performances de grupos feministas, mesas de difusión en el centro de la ciudad, entre otras).

También hemos abordado temas de derechos humanos, como los actos locales por el aniversario del 24 de marzo, actividades del excentro clandestino de detención Pozo 
Soledad Arréguez Manozzo, María Emilia Merlo

Medios y prácticas periodísticas: Instagram como plataforma periodística.

La experiencia de Agencia AUNO

de Banfield, y la casa de Rodolfo Walsh en San Vicente, entre otros. Otro punto fuerte son las historias semanales sobre temáticas o iniciativas de carácter global (por ejemplo, julio 2019 como mes "libre de plástico") y las novedades de cultura digital, como son los cambios en la plataforma Instagram. En los casos mencionados, en primer lugar, hemos pensado los contenidos de forma visual, sabiendo que de todas formas tendrá su versión gráfica. En los últimos casos se trata de temáticas que de otra forma no serían factibles de cubrirse por los rutinas tradicionales de producción de noticias de la agencia. Intentamos constituirnos como un equipo de desarrollo visual periodístico tradicional, y a la vez como un espacio de prácticas para la innovación en temas disruptivos para un medio y espacio de formación.

\section{Conclusiones: primeros aprendizajes y más preguntas}

La incorporación de la plataforma de Instagram al universo digital de Agencia AUNO nos permitió desarrollar de forma orgánica una comunidad de seguidores de nuestra organización, como así también fidelizar a aquellos que ya nos seguían desde otras redes. Además, las funcionalidades de la aplicación nos permitieron humanizar el perfil social del medio, a través de la construcción de una narración periodística con una cronista en cámara.

A su vez entendimos que había mucho potencial en construir una agenda propia, sin desarrollar necesariamente un contenido que tracciona tráfico a la web. En términos periodísticos, estos cuatro años nos llevaron a la necesidad de reestructurar la práctica periodística para "pensar con cabeza de IG", es decir entendiendo la dinámica de la plataforma. Por eso decimos que el equipo de RRSS a cargo de Instagram no son data entry ni community managers, sino que se constituyen como "periodistas visuales", capaces de construir un relato periodístico con base en un material fotográfico o audiovisual. Estos periodistas visuales requieren de una serie de habilidades y aptitudes en torno al uso de redes sociales: redacción periodística, escritura web, escritura SEO, manejo de programas de edición de fotografías y videos, gestión de comunidades en línea, utilización de herramientas de gestión de redes sociales, entre otros.

No quedan dudas que hoy las noticias viajan por diferentes espacios, y que se tejen relatos que se expanden en las reglas y dinámicas de los espacios digitales. Sin embargo, la incorporación del potencial de la narrativa transmedia a las producciones periodísticas aún debe despejar varios interrogantes.

En ese sentido, se requiere de la construcción de relatos periodísticos que puedan producirse con la dinámica de distintas plataformas y medios (extensiones) y que a su vez inviten a la participación de los ciudadanos en la expansión de esas historias. Sin duda este punto arroja varios interrogantes sobre cómo debería ser un newsma- 
Austral Comunicación

Volumen 9, número 1 (Junio de 2020): 1-12. ISSN 2313-9137 E

king transmedia, qué tipo de rutinas periodísticas reclama, y que inversión de recursos requiere.

Asimismo, la certeza del nuevo escenario pone el foco en el comportamiento de las nuevas audiencias y sus hábitos de consumo de noticias. En este punto se plantean dudas sobre cómo interpelar a un público cada vez más fragmentado, sin intermediarios, que buscan contenidos personalizados.

El modo de consumir noticias de las nuevas audiencias, de forma individual y personalizada, también genera incógnitas en torno al rol de los medios en la conversación pública en un escenario hipotético en el que haya carencia de referentes comunes en la comunicación, y una falta de difusión de los temas que todos los ciudadanos deberían estar informados.

Entendemos que estos tiempos requieren desde los medios gestionar los procesos de innovación tecnológica, repensar las lógicas internas de las rutinas laborales, y capacitar a los periodistas en los nuevos lenguajes para contar historias en este entorno digital.

\section{Referencias}

Arréguez Manozzo, S., Fernández, L. (2019). “El día que Internet modificó los medios” en Itinerarios. Caminos hacia la construcción de una idea de comunicación. Lomas de Zamora, Universidad Nacional de Lomas de Zamora (UNLZ).

Campalans C. Renó D., Gosciola V. (2014) Narrativas transmedia entre teorías y prácticas. Bogotá, Editorial Universidad del Rosario.

DeHaan, Gladwell, Murayama, Przybylski, (2013). Motivational, emotional, and behavioral correlates of fear of missing out. Revista Computers in Human Behavior, 29, (4), 1841-1848. Recuperado de https://www.sciencedirect.com/journal/ computers-in-human-behavior/vol/29/issue/4.

DataReportal. (2019). Digital Reports 2019. Recuperado de https://datareportal.com/ reports/digital-2019-global-digital-overview\#: :text=There\%20are\%204.39\%20 billion\%20internet,since\%20this\%20time\%20last\%20year.

De Moraes, D. (2004). El Ecosistema Digital y el Desafío de Democratizar la Red. Revista Razón y Palabra, (37), 2. Recuperado de http://www.razonypalabra.org. $\mathrm{mx} /$ anteriores/n37/dmoraes.html.

Jenkins, H. (2003). Transmedia Storytelling. Technology Review. Recuperado de https:// www.technologyreview.com/s/401760/transmedia-storytelling/.

Orihuela, J. (2002). Nuevos paradigmas de la comunicación. Recuperado de eCuaderno http://www.ecuaderno.com/paradigmas/.

Porto, D. Flores (2012). Reflexiones y técnicas para el ciberperiodista desde los laboratorios de medios interactivos. Madrid, Editorial Fragua. 
Soledad Arréguez Manozzo, María Emilia Merlo

Medios y prácticas periodísticas: Instagram como plataforma periodística.

La experiencia de Agencia AUNO

Ramonet, I. (2011). La explosión del periodismo. Internet pone en jaque a los medios tradicionales. Buenos Aires: Capital Intelectual.

Scolari, C. (2008). Hipermediaciones: Elementos para una teoría de la comunicación digital interactiva. Barcelona: Gedisa.

Van Dijck, J. (2016). La cultura de la conectividad: una historia crítica de las redes sociales. Buenos Aires: Siglo Veintiuno Editores.

We are social, Hoostuite. (2019). Global Digital Report 2019. Recuperado de https:// wearesocial.com/global-digital-report-2019. 